\title{
TMB detection from primary and metastatic lesions should be considered separately: a pan-cancer study.
}

\author{
Xiaoling Shang ${ }^{1}$, Chenglong Zhao ${ }^{2}$, Haining $\mathrm{Yu}^{2}$, and Haiyong Wang ${ }^{2}$ \\ ${ }^{1}$ Shandong Cancer Hospital and Institute \\ ${ }^{2}$ Shandong Cancer Hospital
}

April 27, 2020

\begin{abstract}
Background: In this study, we evaluated the difference in the tumor mutational burden (TMB) score between primary and metastatic lesions in pan-cancer and the different cut-offs for guiding immune checkpoint inhibitors (ICIs) prognosis. Methods: We screened 1661 pan-cancer cases from the cBioPortal database. The Kaplan-Meier method was applied to obtain survival curves that were compared using the log-rank test. The X-tile model was used to determine the optimal cut-off values of TMB. Results: Our results showed that the tissues obtained from the metastatic lesions had more co-occurring gene mutations than the primary lesions $(\mathrm{P}<0.05, \mathrm{Q}<0.05)$. Moreover, tissues from patients with metastatic lesions had a higher TMB score than those from patients with primary lesions $(\mathrm{P}=0.024)$. According to the median cut-off values of TMB from the primary and metastatic lesions, we analyzed the overall survival (OS) in the low TMB and high TMB groups, respectively. The results showed different OS for the two TMB groups in primary and metastatic lesions. Subsequently, we analyzed the optimal cut-off values of TMB score to predict survival in primary and metastatic lesions based on the X-tile model. The optimal cut-off value for the test tissue from primary lesions was $20.19(\mathrm{P}<0.001)$. Importantly, the optimal cut-off value for test tissue from metastatic lesions was 10.18 ( $\mathrm{P}<0.001)$. Conclusion: TMB detection from primary and metastatic lesions should be considered separately to predict survival for ICIs treatment.
\end{abstract}

\section{Introduction}

Treatment with immune checkpoint inhibitors (ICIs) has become extremely popular owing to its high efficacy and long-term benefits. However, only a few patients benefit from ICIs because of the cost of ICIs and adverse events. Therefore, it is important to explore relevant biomarkers. Tumor mutational burden (TMB), which is defined as the number of somatic mutations per DNA mega base (Mb), is one of the emerging prognostic factors to predict the efficacy of immunotherapy, especially ICIs. Clinical studies ${ }^{1-3}$ have indicated that patients with high TMB had better response and survival after the treatment of ICIs in many cancers than those with a low TMB.

TMB is a novel prognostic biomarker of clinical benefit from ICIs independent of the PD-L1 level and is currently being prospectively explored. ${ }^{4,5}$ In clinical practice, the detection of TMB is based on whole exon sequencing or targeted next-generation sequencing of tissue or blood samples. The detected tissues may be from primary or metastatic lesions. Since the number of mutations in primary and metastatic lesions is different, it may have some limitations to use the same standard to predict the prognosis of ICIs for both lesions.

Therefore, in this study, we analyzed the difference in the TMB score between primary and metastatic lesions in pan-cancer and determined the difference in cut-offs for guiding ICIs prognosis.

\section{Methods}


The cBioPortal for Cancer Genomics (https://www.cbioportal.org/) provides genomic and survival data from 1661 tumor-normal pairs from 1661 patients with various cancer types sequenced with MSK-IMPACT assay. ${ }^{6,7}$ Details of tissues processing and MSK-IMPACT analysis have been described in a previous study. ${ }^{8}$ All 1661 patients have received ICIs treatment. We analyzed the number of coexisting mutations and TMB scores in 731 and 930 primary and metastatic lesions, respectively. The overall survival (OS) was calculated from the date of the first ICIs treatment to the time of recent follow-up or death. The Kaplan-Meier method was performed to obtain survival curves that were compared using the log-rank test. Subsequently, the X-tile model was used to determine the cut-off values of TMB. All statistical analyses were performed using Statistical Product and Service Solutions (SPSS) 22.0 software package. The statistical $P$ values were two-sided, and a value of $P<0.05$ was considered statistically significant.

\section{Results}

We analyzed the genomic and survival data of 1661 patients treated with ICIs including 731 and 930 primary and metastatic lesions, respectively. We found that the tissues obtained from the metastatic lesions had more co-occurring gene mutations than those obtained from the primary lesions $(P<0.05, Q<0.05)$ (Figure 1A). Importantly, tissues from patients with metastasis lesions had a higher TMB score than those from patients with primary lesions $(P=0.024)$ (Figure 1B).

The Kaplan-Meier method was applied to analyze the OS. According to the median cut-off values (5.58) of TMB from the primary lesions, we compared the survival in the lesions with low TMB score and the high TMB score. The result revealed that there was no significant difference in OS between the low and high TMB groups in primary lesions $(P=0.416)$ (Figure $2 \mathrm{~A})$. However, using the same cut-off values for TMB from the primary lesions, there was a significant difference in survival between the high TMB and low TMB group in metastatic lesions, and patients with a high TMB had improved survival $(P<0.001)$ (Figure 2B).

To further verify the difference in the effect of TMB scores in guiding the prognosis of ICIs between primary and metastatic lesions, we selected 350 non-small cell lung cancer (NSCLC) patients who were treated with ICIs including 171 and 179 primary and metastatic lesions, respectively. The results of CheckMate227 and CheckMate568 trials ${ }^{9}$ suggested that in NSCLC patients with TMB [?]10 mutations/MB, progression-free survival was significantly prolonged after immunotherapy. Therefore, according to the cut-off value (cutoff=10), we analyzed the survival in NSCLC patients with different TMB scores for primary and metastatic lesions. In patients whose tissue samples came from primary lesions, there was no significant difference in OS $(P=0.474)$ between the low TMB and the high TMB groups (Figure $2 \mathrm{C})$. However, the TMB was significantly associated with OS in patients whose tissue samples came from metastasis lesions $(P=0.012)$ (Figure 2D). This suggested that the role of TMB as a prognostic biomarker for the response to ICIs was different in patients with tissues originated from primary lesions and metastasis lesions.

We predicted the optimal cut-off values of TMB in primary and metastatic lesions in 1661 pan-cancer cases using the X-tile model. The optimal cut-off values of TMB that predicted survival was 20.19 in primary lesions $(P<0.001)$ (Figure 2E) and 10.18 in metastasis lesions $(P<0.001)$ (Figure $2 \mathrm{~F})$. The data revealed that the optimal score of TMB in metastatic lesions was significantly lower than that in primary lesions. Therefore, the optimal cut-off value varied to vary for primary and metastasis lesions in all pan-cancer tissues.

\section{Discussion}

Recent studies indicated that TMB may be a new biomarker to predict the response to ICIs in different tumor entities. ${ }^{10-13}$ Several clinical trials have provided different cut-off values for TMB which leads to difficulties in clinical application. The data providing clinical guidance for TMB cut-off values are scarce. Moreover, the cut-off values of TMB for pan-cancer may not be ideal for clinical use. Therefore, we calculated and compared the difference in TMB cut-offs between primary and metastatic lesions on the basis of cBioPortal mutational data.

In this study, the co-occurring mutations and TMB scores were significantly higher in metastatic lesions than 
in primary lesions. Several theories might explain why metastatic tissues have more gene mutations than primary tissues. First, genetic analysis of matched sets of patients' primary and metastasis lesions revealed that metastatic lesions had mutations common to both and mutations that were distinct from primary lesions, which is the biggest cause of cancer death. Mutations in metastatic lesions were located at the chromosome ends and affected chromosome stability, gene expression regulation, and DNA repair. Previous research indicated that genomic instability was observed in metastatic subclones and/or metastatic lesions of primary lesions. ${ }^{14-17}$ Additionally, studies revealed that new genetic alterations, not seen in primary lesions, were observed in metastasis lesions.

Hong, M. K. H. et al. ${ }^{18}$ revealed that mutation or amplification of both DNA double-strand break repair and mismatch repair gene was indicative of metastasis in prostate cancer progression. Similarly, Brastianos, P. et al. ${ }^{19}$ demonstrated that $53 \%$ of brain metastasis harbored mutations not seen in primary lesions. These data revealed that metastasis lesions would obtain new additional mutations compared to primary lesions, which was consistent with our findings.

Additionally, the variable cut-offs of TMB between primary and metastatic lesions can be attributed to distinct tumor microenvironments and other factors that independently predict response to ICIs including immune infiltration, human leukocyte antigen genotype, clonality, and expression levels of checkpoint molecules. ${ }^{20-23}$ Our result suggested high TMB was associated with the increased OS based on the optimal cut-offs in pan-cancer types. Therefore, TMB as a new biomarker in pan-cancer may reflect fundamental mechanisms that determine the response to ICIs treatment.

Our data addresses a fundamentally important question in ICIs treatment for clinicians. Although TMB could predict survival and response to ICIs across pan-cancer types, the cut-off values of TMB differed between primary and metastatic lesions. Therefore, the TMB score for primary and metastatic lesions should be determined separately. This finding will greatly influence clinical guidance.

This study has a few limitations. This was a retrospective study using data from the cBioPortal database. Therefore, the results should be verified in a prospective study. Furthermore, clarification of appropriate cut-offs of TMB and the integration of relevant clinical variables for each cancer type will be necessary to allow for the application of TMB as a prognostic biomarker to reflect ICIs treatment in future clinical studies.

\section{Data availability statement states}

The data used to support the fifindings of this study are available from the corresponding author upon request.

\section{Ethics Statement}

The present study was approved by the ethics committee of Shandong Cancer Hospital and Institute, Shandong First Medical University and Shandong Academy of Medical Sciences. (Jinan, China).

\section{Author Contributions}

XL S and HY W conceived the study and wrote the paper. CL Z and HN Y performed analyzed the data. All authors approved the manuscript.

\section{Funding}

This study was supported jointly by special funds for Taishan Scholars Project (grant no. tsqn201812149) and Academic Promotion Programme of Shandong First Medical University (2019RC004).

\section{Reference}

1. Rizvi, N. A. et al. Mutational landscape determines sensitivity to PD-1 blockade in non-small cell lung cancer. Science 348, 124-128 (2015).

2. Hugo, W. et al. Genomic and transcriptomic features of response to anti-PD-1 therapy in metastatic melanoma. Cell 165, 35-44 (2016). 
3. Rosenberg, J. E. et al. Atezolizumab in patients with locally advanced and metastatic urothelial carcinoma who have progressed following treatment with platinum-based chemotherapy: a single-arm, multicentre, phase 2 trial. Lancet 387, 1909-1920 (2016).

4. Velcheti V, Kim ES, et al. Prospective clinical evaluation of blood-based tumor mutational burden (bTMB) as a predictive biomarker for atezolizumab (atezo) in 1L non-small cell lung cancer (NSCLC): interim B-F1RST results. J Clin Oncol 2018;36(15_suppl): 12001-01.

5. Hellmann MD, Ciuleanu TE, et al. Nivolumab plus Ipilimumab in lung Cancer with a high tumor mutational burden. N Engl J Med . 2018;378(22):2093-104.

6. Gao, J. et al. Integrative analysis of complex cancer genomics and clinical profiles using the cBioPortal. Sci Signal. 2013 Apr 2;6(269)

7. Robert M. Samstein. Et al. Tumor mutational load predicts survival after immunotherapy across multiple cancer types. Nat Genet.2019 Feb;51(2):202-206.

8. Hugo, W. et al. Genomic and transcriptomic features of response to anti-PD-1

therapy in metastatic melanoma. Cell. 2016 Mar 24;165(1):35-44.

1. Hellmann M D, Ciuleanu T, Pluzanski A, et al. Nivolumab plus Ipilimumab in Lung Cancer with a High Tumor Mutational Burden. The New England Journal of Medicine, 2018, 378(22): 2093-2104.

2. Snyder A, Makarov V, et al. Genetic basis for clinical response to CTLA-4 blockade in melanoma. $N$ Engl J Med 2014;371: 2189-99.

3. Carbone DP, Reck M, et al. First-Line Nivolumab in Stage IV or Recurrent Non-Small-Cell Lung Cancer. N Engl J Med 2017;376: 2415-26.

4. Hellmann MD, Callahan MK, et al. Tumor Mutational Burden and Efficacy of Nivolumab Monotherapy and in Combination with Ipilimumab in Small-Cell Lung Cancer. Cancer Cell 2018;33: 853-61 e4.

5. Rosenberg JE, Hoffman-Censits J, et al. Atezolizumab in patients with locally advanced and metastatic urothelial carcinoma who have progressed following treatment with platinum-based chemotherapy: a single-arm, multicentre, phase 2 trial. Lancet 2016;387: 1909-20.

6. Bojovic, B. \& Crowe, D. L. Dysfunctional telomeres promote genomic instability and metastasis in the absence of telomerase activity in oncogene induced mammary cancer. Mol. Carcinogen. 2013 Feb;52(2):103-17.

7. Vermaat, J. S. et al. Primary colorectal cancers and their subsequent hepatic metastases are genetically different: implications for selection of patients for targeted treatment. Clin. Cancer Res. 2012 Feb 1;18(3):688-99.

8. Yachida, S. et al. Distant metastasis occurs late during the genetic evolution of pancreatic cancer. Nature. 2010 Oct 28;467(7319):1114-7.

9. Roschke, A. et al. Chromosomal instability is associated with higher expression of genes implicated in epithelial-mesenchymal transition, cancer invasiveness, and metastasis and with lower expression of genes involved in cell cycle checkpoints, DNA repair, and chromatin

maintenance. Neoplasia. 2008 Nov;10(11):1222-30.

1. Hong, M. K. H. et al. Tracking the origins and drivers of subclonal metastatic expansion in prostate cancer. Nat. Commun.2015 Apr 1;6:6605.

2. Brastianos, P. et al. Genomic characterization of brain metastases reveals branched evolution and potential therapeutic targets. Cancer Discov. 2015 Nov;5(11):1164-1177.

3. Rooney, M. S., Shukla, S. A., et al. Molecular and genetic properties of tumors associated with local immune cytolytic activity. Cell. 2015 Jan 15;160(1-2):48-61.

4. Balachandran, V. P. et al. Identifcation of unique neoantigen qualities in long-term survivors of pancreatic cancer. 2017 Nov 23;551(7681):512-516.

5. Topalian, S. L. et al. Safety, activity, and immune correlates of anti-PD-1 antibody in cancer. N. Engl. J. Med. 2012 Jun 28;366(26):2443-54.

6. McGranahan, N. et al. Clonal neoantigens elicit $\mathrm{T}$ cell immunoreactivity and sensitivity to immune checkpoint blockade.Science, 2016 Mar 25;351(6280):1463-9. 


\section{Figure legends.}

Figure 1. The TMB comparison between primary and metastasis. A: The coexisting gene mutation in metastasis lesions (blue point) was more than that in primary tumors (gray point) $(P<0.05, Q<0.05)$. B: The TMB score comparison between 731 primary cases and 930 metastasis cases $(P=0.024)$.

Figure 2. Effect of tumor mutational burden on survival after ICIs treatment. A: Survival curves for patients whose tissues from the primary tumors between the high TMB $(\mathrm{n}=357)$ and the low TMB $(\mathrm{n}=369)$ basing on the median cutoff value $(5.58)(P=0.486)$. B: Survival curves for patients whose tissues from the metastasis lesions between the high TMB $(\mathrm{n}=539)$ and the low TMB $(\mathrm{n}=389)$ basing on the median cutoff value $(P<0.001)$. C: Survival curves for NSCLC patients whose tissues from the primary tumors between the high TMB $(\mathrm{n}=49)$ and the low TMB $(\mathrm{n}=122)$ basing on the cutoff value $=10(P=$ 0.474). D: Survival curves for NSCLC patients whose tissues from the metastasis lesions between the high TMB $(\mathrm{n}=68)$ and the low TMB $(\mathrm{n}=111)$ basing on the cutoff value $=10(P=0.012)$. E: Survival curves for patients whose tissues from the primary tumors between the high TMB $(\mathrm{n}=73)$ and the low TMB $(\mathrm{n}$ $=653)$ basing on the optimal cutoff value $=20.19$ in pan-cancer types $(P<0.001)$. F: Survival curves for patients whose tissues from the metastasis lesions between the high TMB $(\mathrm{n}=289)$ and the low TMB $(\mathrm{n}=$ $639)$ basing on the optimal cutoff value $=10.82$ in pan-cancer types $(P<0.001)$.

Figure 1.

A

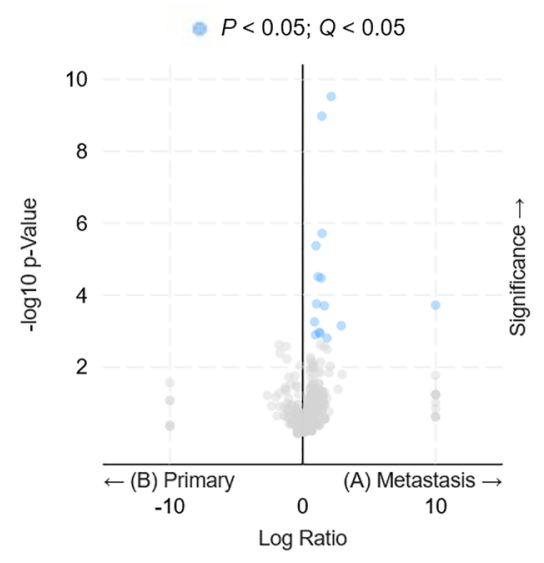

B Primary:10.79 $\pm 0.7001(\mathrm{n}=731)$

Metastasis: $12.89 \pm 0.6042(n=930)$

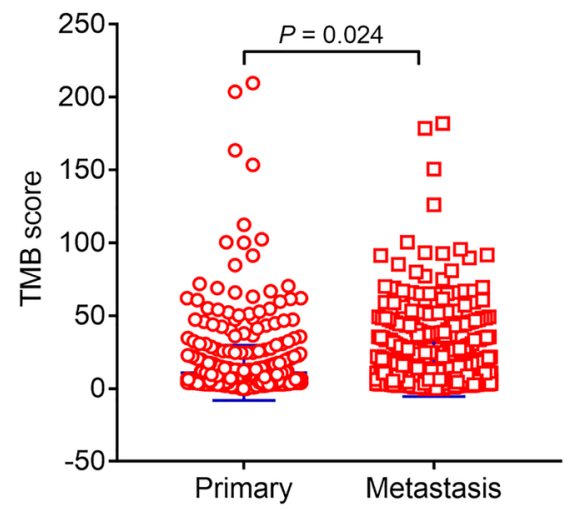

Figure 2. 
A

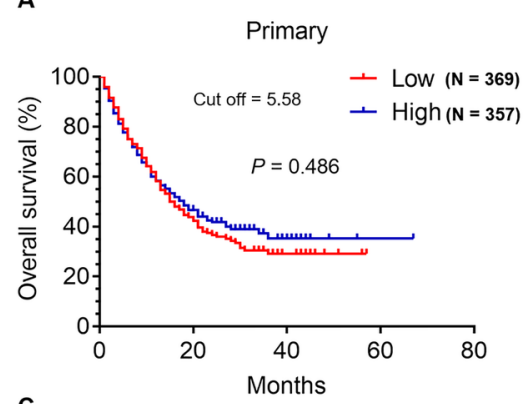

C

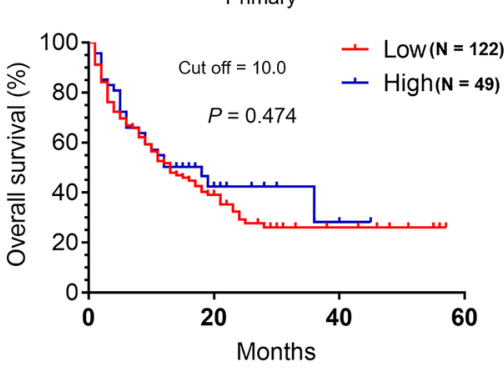

E

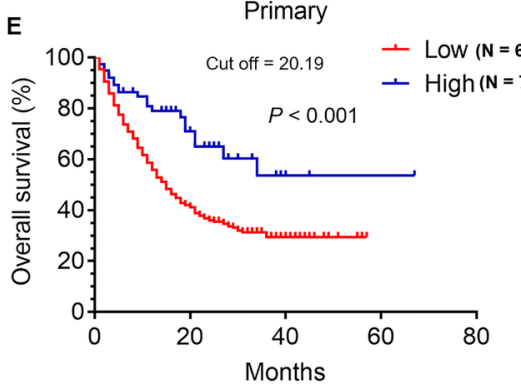

B

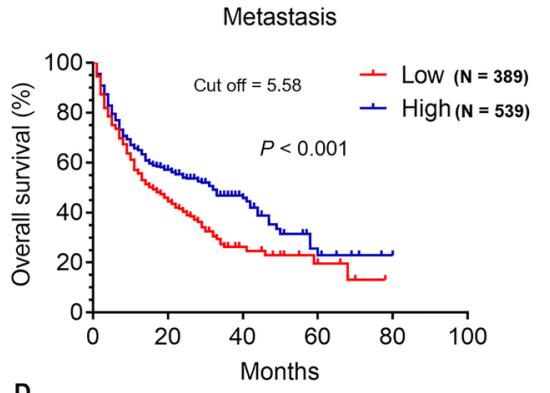

D

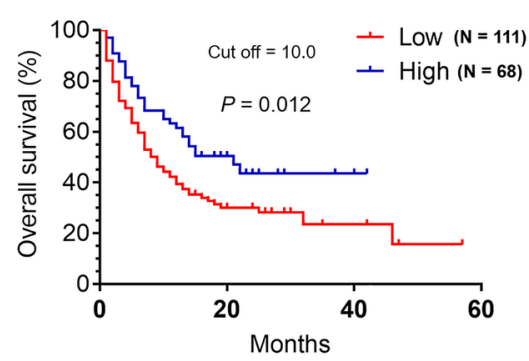

F

Metastasis

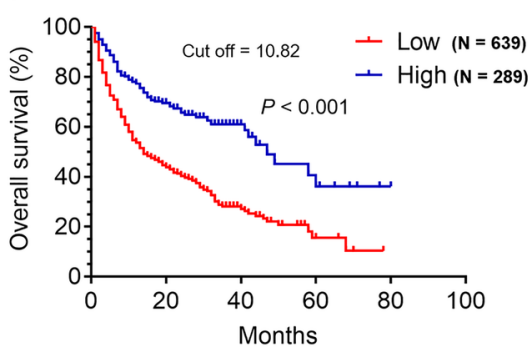

